\title{
An Educational Training Kit for an Efficient GPS Anti- Jamming System
}

\author{
https://doi.org/10.3991/ijet.v16i06.21251
}

Sarmad Ahmed Shaikh ${ }^{(凶)}$

Sindh Madressatul Islam University, Karachi, Pakistan

sarmadesmiu.edu.pk / sarmad.ahmed107@gmail.com

Muhammad Minhaj Arshad

Karachi Institute of Economics and Technology, Karachi, Pakistan

\section{Hammad M. Cheema}

Research Institute for Microwave and Millimeter-Wave Studies (RIMMS), National University of Sciences and Technology (NUST), Islamabad, Pakistan

Muhammad Zainul Abideen, Syed Masaab Ahmed Karachi Institute of Economics and Technology, Karachi, Pakistan

\begin{abstract}
Global positioning system (GPS) is one of the key satellite-based radio technology providing navigational services on earth or near earth. The increasing usage of GPS applications in daily life has proved that students/researchers of many educational departments including information and communication technology (ICT) are particularly interested to learn and visualize the GPS anti-jamming techniques in a simpler way in their major communication courses. It is a fact that GPS radio signal is vulnerable in various ways to jamming signals launched by malicious elements. The traditional available GPS anti-jamming techniques generally involve either added hardware and/or computational complexity which introduce difficulty for students to understand the basic concepts. As an alternative, this paper presents a simpler GPS antijamming system kit based on L1-band $(1.575 \mathrm{GHz})$ of GPS for education purpose. More specifically, the proposed antenna array, integrated with two couplers, cancels out the jamming signal in radio frequency (RF) domain using sum and difference patterns generated by the latter. The simulated and measured results, in terms of scattering parameters and power profiles, of the proposed system have shown that the jamming signal can be eliminated with less complexity. Eventually, the proposed GPS anti-jamming system has great potential to be used in ICT related disciplines for learning and training purposes.
\end{abstract}

Keywords - Global positioning system, Learning, ICT, Antenna arrays 


\section{Introduction}

The scope of emerging technologies in learning is increasing day-by-day where many researchers come under a common goal and start to develop the solutions of the problems specially related to engineering education. This approach of collaboratively learning and development particularly can be helpful in enhancing the engineering labs infrastructure and human skills [1]. Bearing this in mind, this paper is dealing with a global positioning system (GPS) training kit design which can be used as a learning tool for information and communication technology (ICT) students/researchers.

The GPS, initially designed for military purposes, provides geo-location and navigation information from more than 20,000 kilometers above the earth using approximately 24 satellite systems. Nowadays, it provides uninterruptable services to a wide range of application scenarios from military to civilian organizations using frequency bands L1/L2/L5 (1.575/1.227/1.176 GHz) [2]. Currently, GPS is being used in industrial sector wherever information regarding time, location, heading and velocity is desired $[3,4,5]$. The well-known applications of GPS include positioning, navigation, surveying, security, geographic mapping, television signals broadcasting, aviation, and surveillance and so on. Since 2000, GPS has been used actively in several civilian sectors. However, a civilian GPS receiver, as compared to military GPS receiver, is more vulnerable to jamming signals because all the signal characteristics (e.g., code, frequency, and modulation method) are open to public [6]. Thus, in various courses related to ICT discipline, students/researchers are generally interested to learn the basic functionality of GPS operations including jamming and anti-jamming techniques.

A GPS jammer is a self-contained transmitter device used to jam the original GPS signal by sending radio signals of the same frequency i.e., L1-band $(1.575 \mathrm{GHz})$, with high signal power e.g., greater than $56 \mathrm{~dB}$ from the original GPS signal ( -120 dBm). Once this occurs, the GPS receiver becomes unable to perform its normal functionality. Thus, it is necessary to study and to develop anti-jamming techniques to provide continuous GPS signal access to the devices.

This paper presents a simple approach for GPS anti-jamming which could be easily understood and used by fresh ICT students in their major communication courses. The proposed approach consists of patch antenna array and coupler, designed at 1.575 GHz GPS band. The rest of the paper is organized in the following manner. Section 2 and 3 present the literature review and the proposed methodology, respectively. The simulation and measurement results observed and experimental setups (with and without jamming sources) assumed, are provided in section 4. Finally, we conclude the paper in Section 5 .

\section{Background and Literature Review}

To explore the effects of GPS jamming and its mitigation concepts, various studies and educational tools/kits have been proposed in literature which are mainly related to 
digital signal processing and antenna array processing techniques. For instance, an anti-jamming GPS receiver that removes the jamming effects and preserves the GPS signal phase continuity is proposed in [7]. However, the proposed algorithm is highly complex in terms of computation. Similarly, interference produced by jamming to the GPS signals can be eliminated by using adaptive signal processing [8, 9]. These methods require jammer synthesis based on time-frequency distributions and the performance is compromise between antenna array size and computational complexity. More specifically, the techniques like adaptive array processing allow exploitation of the spatial domain for further improved anti-jamming and signal protection [9-11] with additional burden of high dimensional signal processing matrices. In a slightly different approach, a statistical space-time null widening (SSTNW) method has been proposed in [10] which suppresses interferences effectively in high dynamic conditions and is able to remove disturbance by non-ideal factors. In the antenna-based approaches, pattern and polarization diversity to enable null-steering has been proposed in [11] that suppresses up to two jamming signals incident from the angular region close to horizon. It introduces additional hardware complexity on the frontend. Similarly, [12] presents a high-temperature stable antenna array applied to an adaptive anti-jamming system in satellite navigation. Additionally, in [13], a 5element antenna array-based GPS anti-jamming technique is proposed which is able to provide nulls in two directions at the cost of increased hardware.

As evident, majority of the reported anti-jamming approaches either require sophisticated signal processing algorithms or complex hardware structures which increase the computational and/or hardware burden in the GPS receivers. Eventually, it becomes somehow difficult for fresh university students from ICT related disciplines to learn the basic concepts related to GPS anti-jamming. As an alternative, this paper presents a simple and easy to understand approach for the GPS anti-jamming which can be implemented in the radio frequency (RF) domain. Utilizing an array of two antennas and associated couplers, the sum and difference patterns of the incoming RF signal are combined in a way to suppress the high-power jamming signal without deteriorating the lower power GPS signal.

\section{$3 \quad$ Proposed Methodology}

The proposed approach for GPS anti-jamming in RF domain, as shown in Fig. 1, includes an array of two microstrip patch antennas integrated with two $180^{\circ}$ hybrid rat race couplers (ring couplers) and a reference GPS signal. The combination, working at $\mathrm{L} 1$ frequency band $(1.575 \mathrm{GHz})$, generates the sum and difference pattern of the incoming input signal by introducing $0^{\circ}$ and $180^{\circ}$ phase differences, respectively. More specifically, the received jamming plus GPS signal on the antenna array is processed in such a way that one of the antenna elements (i.e., Antenna 1) signal is sent towards first coupler's input port. While, on other input port, we provide a GPS reference signal to obtain the jamming signal only on the difference port (delta port $-\Delta_{1}$ ). Furthermore, the second antenna element's signal (jamming plus GPS) and the jamming signal coming from the first coupler's delta port are provided as input to the 
second coupler. Thus, the original GPS signal is easily extracted at delta port $\left(\Delta_{2}\right)$ of coupler 2 utilizing simple arithmetic implemented through couplers. In this way, the high amplitude jamming signal can be removed and the original GPS signal after recovering can be made available for further processing without (saturating) the GPS receiver. The design of antenna array and coupler are described below.

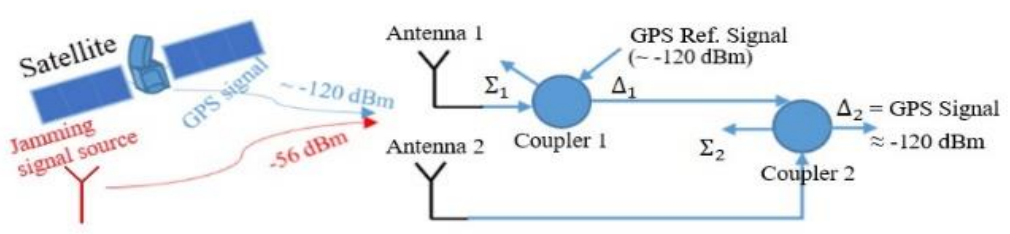

Fig. 1. Block diagram of the proposed GPS anti-jamming technique

a) Antenna array design

The microstrip patch antenna (MPA) is a popular antenna because of the ease of fabrication on the printed circuit board (PCB). Several configurations can be used to feed microstrip patch antennas such as probe feed, microstrip edge feed with inset transmission line (TL), and quarter wave transformer matched TL $[14,15]$. In this work, an inset fed MPA array of two elements separated by $0.5 \lambda$ distance, at L1 band using FR4 substrate $\left(\epsilon_{-} \mathrm{r}=4.5, \mathrm{~h}=1.6 \mathrm{~mm}\right.$, and tangent loss $\left.=0.02\right)$ has been designed. The simulated antenna array is shown in Fig. 2 (a) with all the design parameters. The obtained simulation results show that the array resonates at $1.575 \mathrm{GHz}$ frequency with return loss (S11) parameter of $-21.45 \mathrm{~dB}$, as shown in Fig. 2 (b). Moreover, the directivity obtained is $7.55 \mathrm{dBi}$. Furthermore, the fabricated MPA was tested using vector network analyzer (VNA). The measured scattering parameter (S11) is illustrated in Fig. 2(b), which shows that the fabricated antenna array resonates at $1.61 \mathrm{GHz}$ with return loss of $-12.7 \mathrm{~dB}$. 


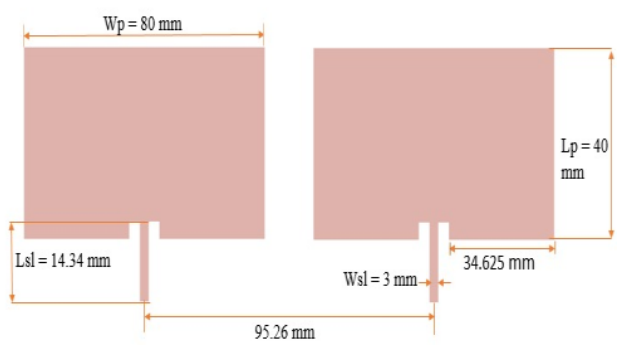

a) Simulated MPA array

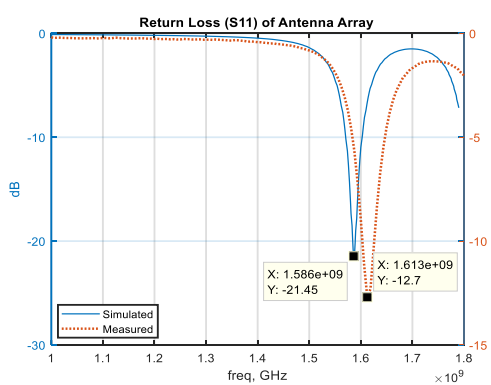

b) simulated and measured return loss (S11) versus frequency of the MPA array

Fig. 2. Antenna array design

b) Coupler design

The $180^{\circ}$ hybrid rat race (HRR) coupler or ring coupler, is a type of coupler used in $\mathrm{RF} /$ microwave devices, generally consisting of four ports. This component offers both in-phase (sum $-\Sigma$ ) and out-phase (difference $-\Delta$ ) relationship at the designated output ports. For more details about ring coupler's usage and realization, readers are referred to $[16,17,18]$. Thus, in this work, the coupler has been used to obtain the $\Sigma$ and the $\Delta$ of the input signal. The ring coupler has been designed at $1.575 \mathrm{GHz}$. The designed coupler using FR4 substrate is shown in Fig. 3 (a). The simulated return loss scattering parameters (S11, S22, S33, and S44) of all four ports are illustrated in Fig. 3 (b) which show that all ports resonate at central frequency with return loss approximately -47 dB. Moreover, Fig. 3 (c) and (d) shows the simulated coupler's phase differences for $\sum\left(0^{0}\right)$ and $\Delta$ ports $\left(180^{\circ}\right)$, respectively. Furthermore, the same coupler was fabricated and characterized using vector network analyzer (VNA). The measured scattering parameters and phase differences are shown in Fig. 3 (e) to Fig. 3 $(\mathrm{g})$, showing good correlation with the simulated results. 


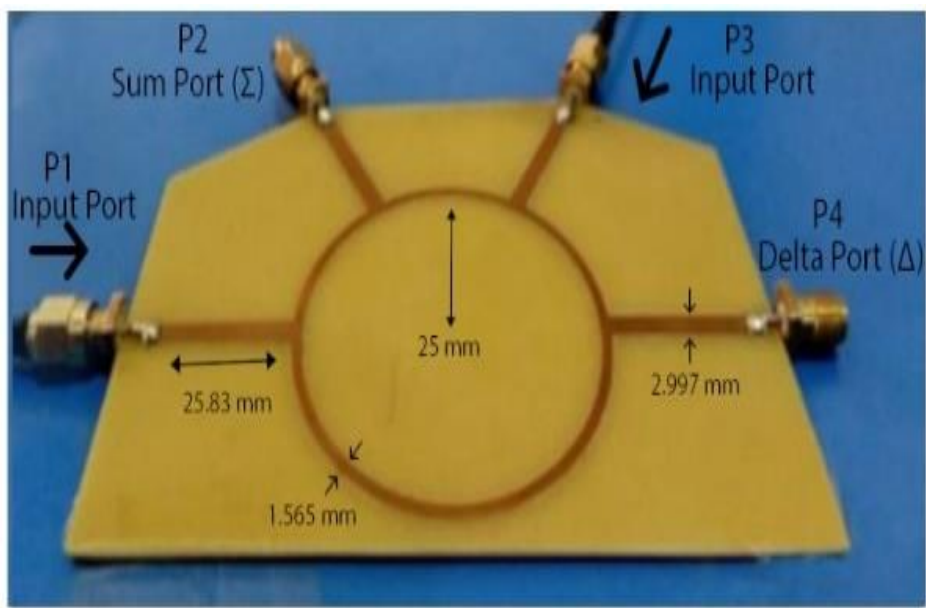

a). Fabricated coupler with design parameters

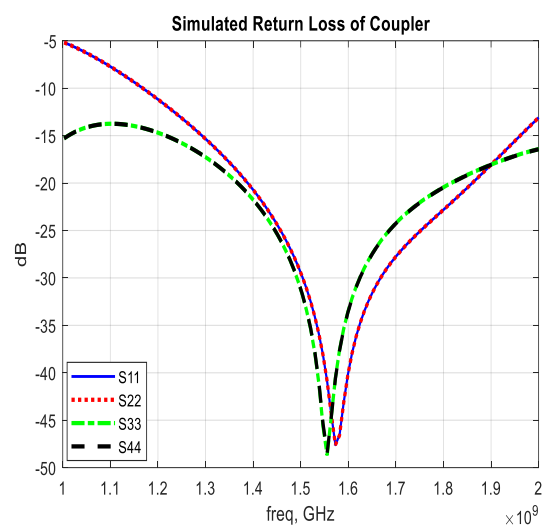

b). Simulated return loss of coupler.

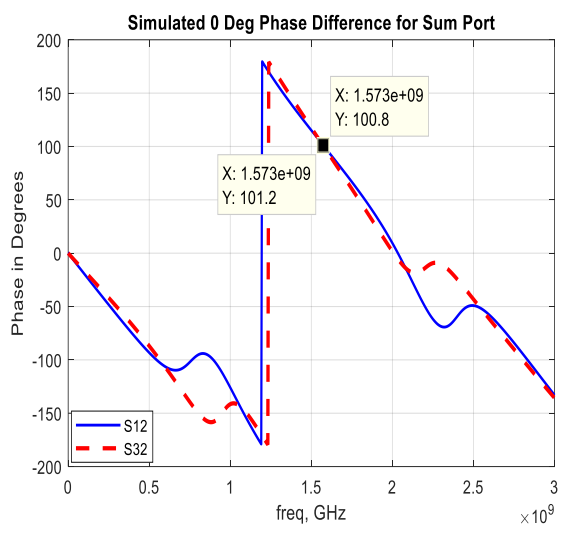

c). Simulated scattering (phase) parameters of ring coupler for $\sum$-port. 


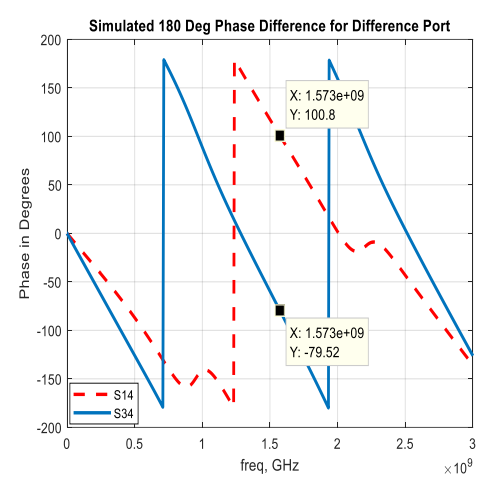

d). Simulated scattering (phase) parameters of ring coupler for $\Delta$-port.

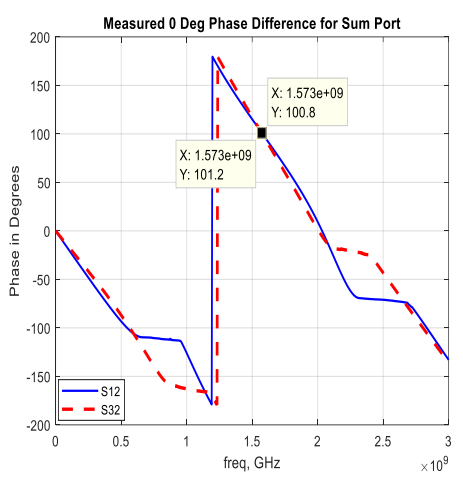

f). Measured scattering (phase) parameters of ring coupler for $\sum$-port.

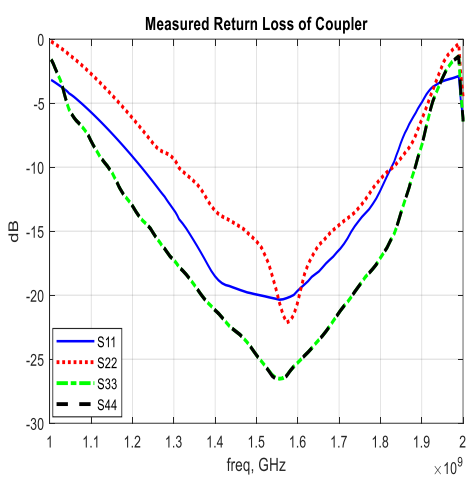

e). Measured return loss of coupler.

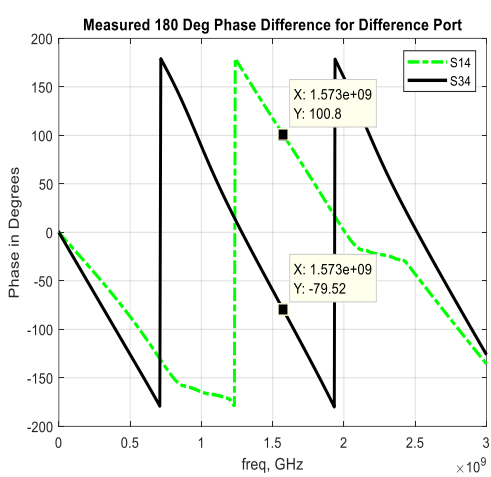

g). Measured scattering (phase) parameters of ring coupler for $\Delta$-port.

Fig. 3. Various simulated and measured results of the designed coupler.

\section{$4 \quad$ Results and Discussions}

In this section, the results of the proposed design shown in Fig. 4 (a) are discussed. Fig. 4 (b) shows the simulated scattering parameters of the reference port of coupler 1 and delta (difference) port of coupler 2. Both desired ports resonate at $1.575 \mathrm{GHz}$ and provide return loss of $-22.65 \mathrm{~dB}$ for reference port and $-30.54 \mathrm{~dB}$ for difference port. Similarly, Fig. 4 (b) also illustrates the measured return loss of the fabricated circuit which is slightly shifted to $1.615 \mathrm{GHz}$ and provides return loss $-12.4 \mathrm{~dB}$ for reference port and $-15.2 \mathrm{~dB}$ for delta port. Furthermore, as shown in Fig. 5 (a), the fabricated 
circuit was measured in anechoic chamber to validate the performance for antijamming. In this regard, initially, a signal generator was used to generate L1 band signal on transmission side with transmission power $\left(P_{t}\right)$ of $0 \mathrm{dBm}$ (transmission antenna gain of $7 \mathrm{dBi}$ ) and measured the received power $\left(P_{r}\right)$ as $-49.08 \mathrm{dBm}$ on receiving side, as shown in Fig. 5 (b), by employing a single element patch antenna. This $P_{r}$ is measured as a reference power to be achieved after employing the proposed circuit for both with and without jamming. Further, in this work, two experimental setups have been considered. In experiment one, we consider no any jamming source while in experiment two we consider jamming source and test the proposed system in anechoic chamber. Both experiments are discussed below with observed results.

First, the fabricated circuit was tested without employing any jamming source. In this regard the measurement setup shown in Fig. 6(a) was utilized. All the parameters of transmitter side are considered the same as defined earlier and all other ports were terminated using $50 \Omega$ loads. The measured received power at port 4 of coupler 2 is $46.04 \mathrm{dBm}$, as illustrated in Fig. 6 (b), which is close to the required reference received power $-49.08 \mathrm{dBm}$. Hence, the circuit is working normal when no jamming source is involved.

Next, the same circuit was measured in the presence of the jamming source. Whereas, in the transmission side, two horn antennas were excited with unequal input powers using $3 \mathrm{~dB}$ attenuator (one antenna for GPS source signal with low transmission power and another antenna for jamming source signal with high transmission power). In this way, the desired circuit, deployed as receiving array, measured the received power as $-52.94 \mathrm{dBm}$, as illustrated in Fig. 6 (b), which is again in good match to the original received reference signal i.e., $-49.08 \mathrm{dBm}$. Thus, it is certain that the proposed circuit works appropriately and cancels out the high-power jamming signal and helps to avoid the GPS receiver to be saturated by jamming source.

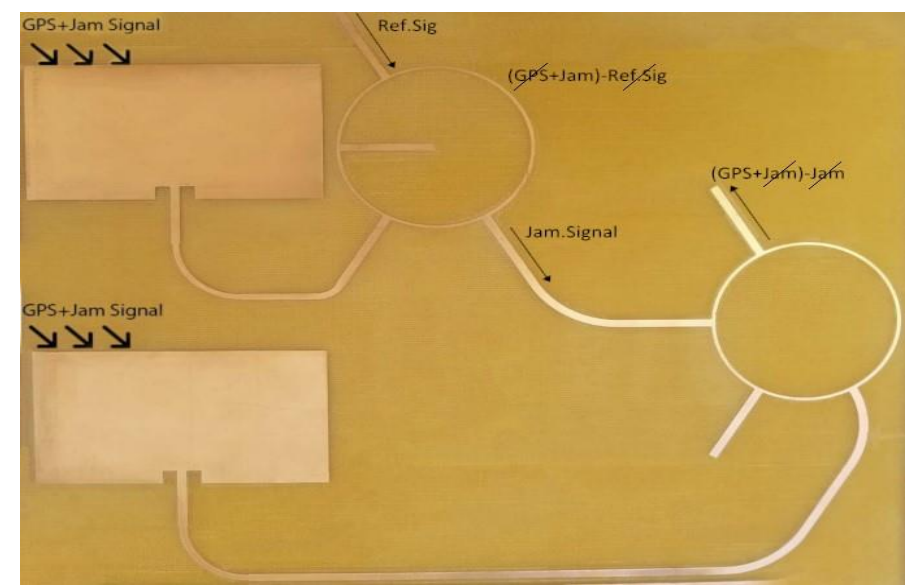

a). Fabricated circuit 

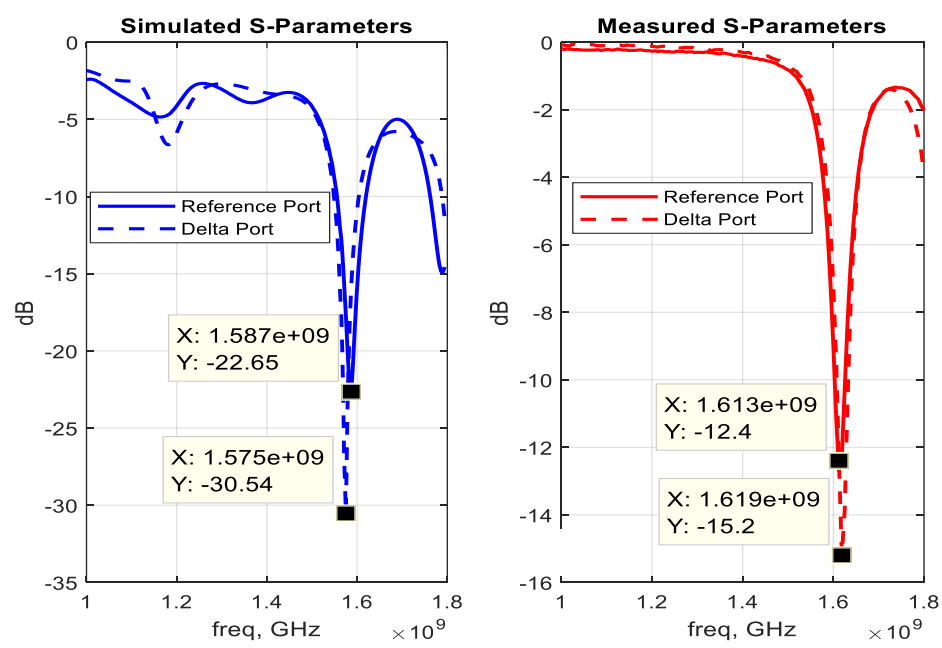

b). Simulated and measured return loss of designed circuit's reference (Coupler1) and delta ports (Coupler2).

Fig. 4. The proposed circuit design for GPS anti-jamming

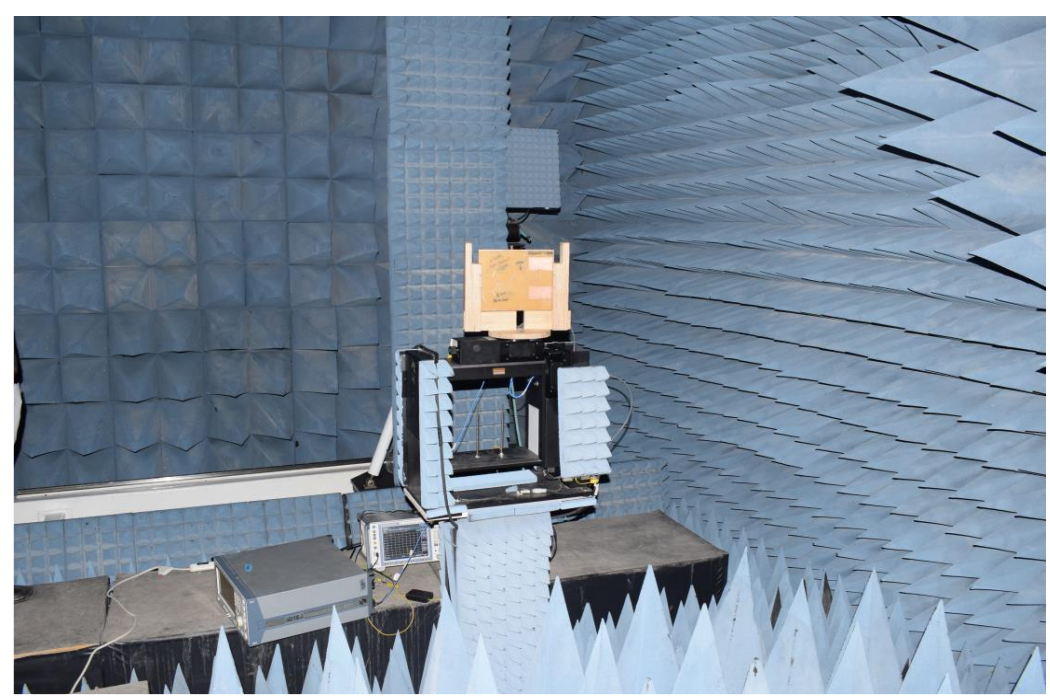

a). Measurement setup in anechoic chamber 


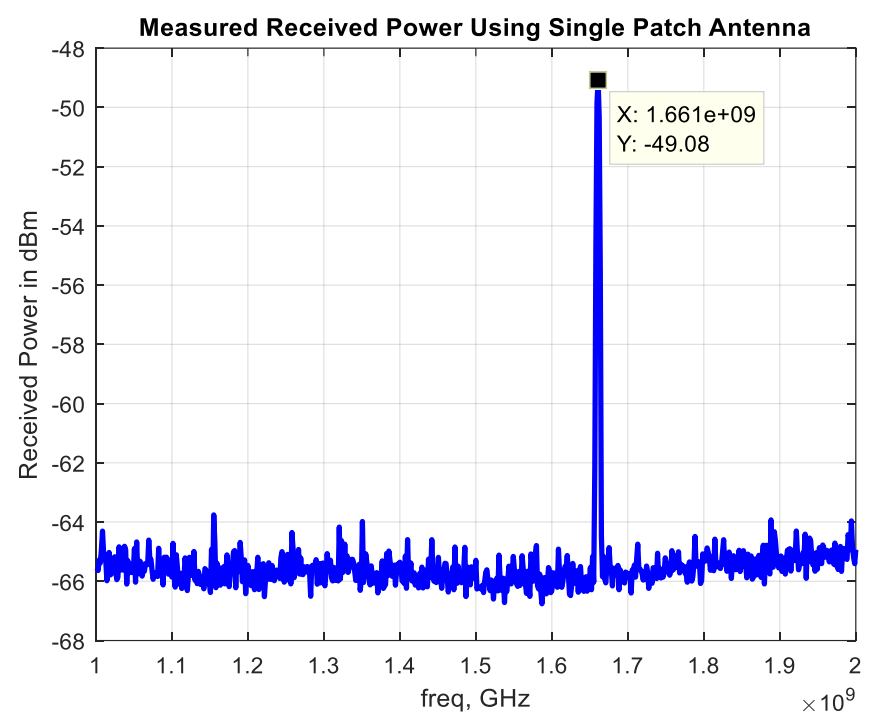

b). Received power using single patch antenna.

Fig. 5. Experimental testing

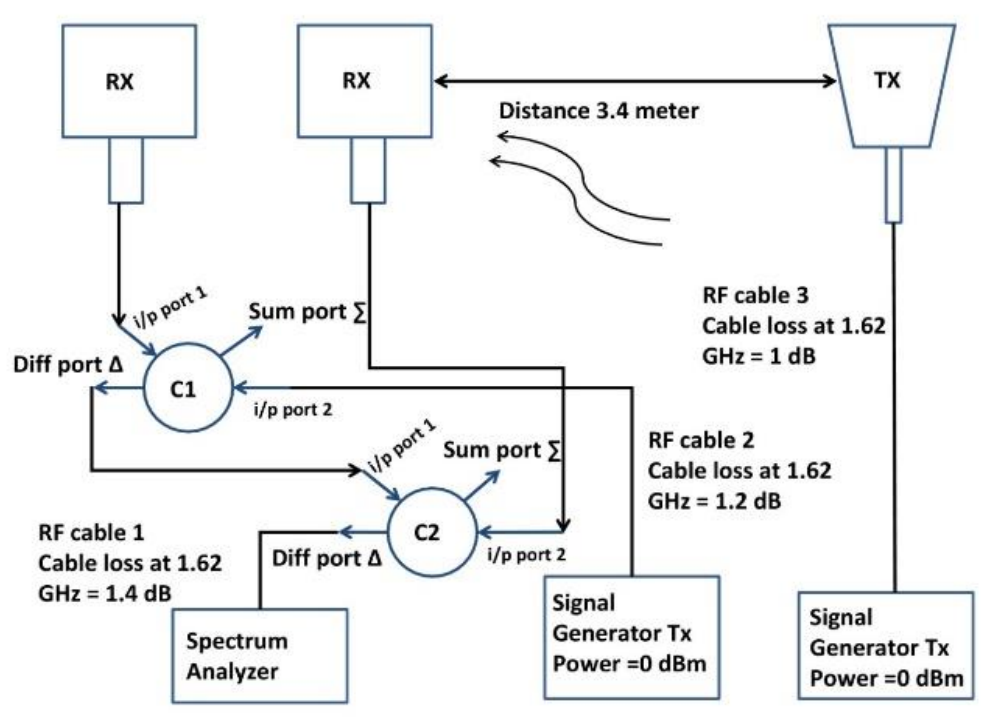

a). Experimental setup 


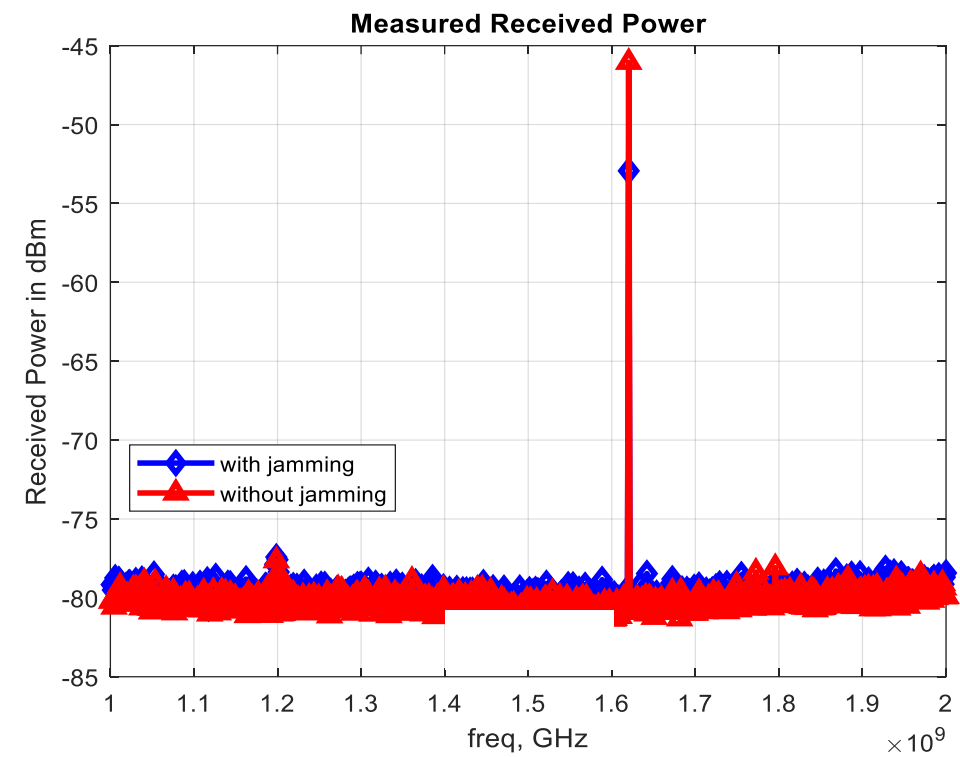

b). Received measured power with (diamond) and without (triangle) jamming.

Fig. 6. Proposed circuit's testing

\section{Conclusion}

In this paper, a simple RF domain-based GPS anti-jamming approach has been proposed as an educational technology for learning and research. The proposed system, consisting of patch antennas array and couplers, is capable of working in both scenarios of with and without jamming source. It performs the anti-jamming without using any complex structure and saving the GPS receiver from going into the saturation due to high power of jamming source. The proposed method can be utilized to design anti-jamming systems for other GPS bands as well. Thus, the proposed approach has a great potential to be used for learning and research purpose for ICT students/researchers which will enable them to learn the basic principles of GPS antijamming in a simpler way.

\section{Acknowledgement}

The authors wish to acknowledge the Higher Education Commission (HEC) of Pakistan for the financial support for this research work under Startup Research Grant Program scheme with reference No: 21- 2372/SRGP/R\&D/HEC/2019. 


\section{$7 \quad$ References}

[1] E. A. Gamie, S.A. El-Seoud, and M.A. Salama, "Comparative Analysis for Boosting Classifiers in the Context of Higher Education," International journal of Emerging Technologies in Learning (iJET), vol. 15, no. 10, pp. 16-26, 2020. https://onlinejournals.org/index.php/i-jet/article/view/13663/7089. https://doi.org/10.3991/ijet.v15i10. 13663

[2] E. Kaplan and C. Hegarty, Understanding GPS: Principles and Applications, London: Artech House, 2006.

[3] M. L. Psiaki, B. W. O'Hanlon, J. A. Bhatti, D. P. Shepard and T. E. Humphreys, "GPS Spoofing Detection via Dual-Receiver Correlation of Military Signals," in IEEE Transactions on Aerospace and Electronic Systems, vol. 49, no. 4, pp. 2250-2267, 2013. https://ieeexplore.ieee.org/document/6621814. https://doi.org/10.1109/taes.2013.6621814

[4] Z. Haider and S. Khalid, "Survey on effective GPS spoofing countermeasures," Sixth International Conference on Innovative Computing Technology (INTECH), Dublin, pp. 573577, 2016. https://ieeexplore.ieee.org/document/7845038. https://doi.org/10.110 9/intech.20167845038

[5] S. Massab, M. Z. Abideen, M. M. Arshad, and S. A. Shaikh, "Collaboratively Learning and Developing a Tool Kit Circuit for GPS Anti-Jamming," in Springer - 22nd International Conference on Interactive Collaborative Learning (ICL), Bangkok, Thailand, pp. 12321242, 09 2019. https://link.springer.com/chapter/10.1007\%2F978-3-030-40274-7_23. https://doi.org/10.1007/978-3-030-40274-7_23

[6] H.S. Kim, B.G. Kim, S.W. Moon, S.H. Kim, S.H. Jung, S.G. Kim, and Y.S. Eo, "Design of a High Dynamic-Range RF ASIC for Anti-jamming GNSS Receiver," in Journal of Positioning, Navigation, and Timing, vol. 3, no. 3, pp. 115-122, 2015. http://koreascience.or.kr/article/JAKO201530848413725.page. https://doi.org/10.11003 lipnt.2015.4.3.115

[7] Y. D. Zhang and M. G. Amin, "Anti-Jamming GPS Receiver with Reduced Phase Distortions," in IEEE Signal Processing Letters, vol. 19, no. 10, pp. 635-638, 2012. https://ieeexplore.ieee.org/document/6247468. https://doi.org/10.1109/1sp.2012.2209873

[8] Y. Zhang, M. G. Amin and A. R. Lindsey, "Anti-jamming GPS receivers based on bilinear signal distributions," MILCOM Proceedings Communications for Network-Centric Operations: Creating the Information Force (Cat. No.01CH37277), vol. 2, pp. 1070-1074, 2001. https://ieeexplore.ieee.org/document/986006. https://doi.org/10.1109/milcom.2001.986006

[9] M. G. Amin and Y. Zhang, "Interference suppression in spread spectrum communication systems," in The Wiley Encyclopedia of Telecommunications, J. G. Proakis, Ed. Hoboken, NJ: Wiley 2002. https://doi.org/10.1002/0471219282.eot224

[10] B. Zhang, H. Ma, X. Sun, Q. Tan and H. Pan, "Robust anti-jamming method for high dynamic global positioning system receiver," in IET Signal Processing, vol. 10, no. 4, pp. 342-350, 2016. https://ietresearch.onlinelibrary.wiley.com/doi/full/10.1049/ietspr.2015.0122. https://doi.org/10.1049/iet-spr.2015.0122

[11] N. Rezazadeh and L. Shafai, "A Compact Antenna for GPS Anti-Jamming in Airborne Applications," in IEEE Access, vol. 7, pp. 154253-154259, 2019. https://ieeexplore.ieee.org/document/8879485.https://doi.org/10.1109/access.2019.294882 $\underline{3}$

[12] Y. Liu, S. Zhang and Y. Gao, "A High-Temperature Stable Antenna Array for the Satellite Navigation System," in IEEE Antennas and Wireless Propagation Letters, vol. 16, pp. 1397-1400,2017.https://ieeexplore.iee.org/document/7782420. https://doi.org/10.1109 /lawp.2016.2639068 
[13] K. Wu, L. Zhang, Z. Shen and B. Zheng, "An Anti-Jamming 5-Element GPS Antenna Array Using Phase-Only Nulling," in 6th International Conference on ITS Telecommunications, Chengdu, pp. 370-373, 2006. https://ieeexplore.ieee.org/document/4068606. https://doi.org/ 10.1109/itst.2006.288915

[14] W. L. Stutzman and G. A. Thiele, Antenna Theory and Design, 2nd Edi, John Wiley \& Sons Inc, Newyork, 1998.

[15] D. M. Pozar, Microwave Engineering, 3rd Edi, John Wiley \& Sons, New York, 2005.

[16] S. A. Shaikh and A. M. Tonello, "Performance Analysis of 180-degree HRR Coupler Used for Direction Finding with an Antenna Array," in International Journal of Biomedical \& Online Engineering (iJOE), vol. 13, no. 10, pp. 86-102, 2017. https://onlinejournals.org/index.php/i-joe/article/view/7410. https://doi.org/10.3991/ijoe.v13i10.7410

[17] M. L. Chuang, "Miniaturized ring coupler of arbitrary reduced size," in IEEE Microwave and Wireless Components Letters, vol. 15, no. 1, pp. 16-18, 2005. https://ieeexplore.ieee.org/document/1377338. https://doi.org/10.1109/lmwc.2004.840960

[18] K. W. Eccleston and S. H. M. Ong, "Compact planar microstripline branch-line and ratrace couplers," in IEEE Transactions on Microwave Theory and Techniques, vol. 51, no. 10, pp. 2119-2125, 2003. https://ieeexplore.ieee.org/document/1234753. https://doi.org/10.1109/tmtt.2003.817442

\section{Authors}

Dr. Sarmad Ahmed Shaikh was born in Pakistan. He received the Bachelor's Degree in Telecommunication Engineering from FAST-NUCES, Pakistan, in 2011, the M.S. degree in Electronics Engineering from Sabanci University, Istanbul, Turkey, in 2015, and the Ph.D. degree in Information Technology from the University of Klagenfurt, Klagenfurt, Austria, in 2018. He is currently an Assistant Professor with the Faculty of Information Technology, Sindh Madressatul Islam University (SMIU), Karachi, Pakistan. His research interests include RF/microwave engineering, radio source localization, antenna arrays designing, and wireless sensor networking (IoT) \& artificial intelligence.

Dr. Hammad M. Cheema (SM IEEE) received Bachelors in Electrical Engineering from NUST, Pakistan, MSc in Telecommunications from Technical University of Denmark and $\mathrm{PhD}$ in Electrical Engineering from Eindhoven University of Technology, The Netherlands. His $\mathrm{PhD}$ research was related to integrated circuit design of phase locked loops and millimeter wave components for $60 \mathrm{GHz}$ broadband wireless transceivers. From 2011 to 2013, Dr. Cheema was a post-doctoral researcher at the IMPACT Lab at KAUST, Saudi Arabia working on $60 \mathrm{GHz}$ transmitter chips and onchip antennas. Dr. Cheema also worked on flexible and printed electronics for lowcost inkjet printed applications using paper and plastic substrates. Currently, Dr. Cheema is an Associate Professor and Principal Research Institute for Microwave and Millimeter-Wave Studies (RIMMS) at NUST, Pakistan working on MMICs, RF Systems, power amplifiers and IOT applications. Dr. Cheema's industrial experience includes various technical roles at Motorola \& Ericsson in Pakistan, and Danske Telecom, Accenture and Microsoft in Denmark. He has authored more than 50 journal and 
conference papers, one book and holds a US patent to his name. He was awarded the best university teacher award in 2015 and the best Institute researcher award in 2019.

M. Minhaj Arshad was born in Pakistan. He received bachelor's degree in Avionics Engineering from PAF-KIET (Karachi Institute of Economics \& Technology), Karachi, Pakistan in 2019. His research interests include antenna design and microwave engineering.

M. Zainul Abideen was born in Pakistan. He received bachelor's degree in Avionics Engineering from PAF-KIET, Karachi, Pakistan in 2019. His research interests include antenna design and microwave engineering.

Syed Masaab Ahmed was born in Pakistan. He received bachelor's degree in Avionics Engineering from PAF-KIET, Karachi, Pakistan in 2019. His research interests include antenna design and microwave engineering.

Article submitted 2021-01-16. Resubmitted 2021-02-27. Final acceptance 2021-02-29. Final version published as submitted by the authors. 